\section{Pleuramesotheliom: Nintedanib ohne Überlebensvorteil}

Scagliotti GV et al. Nintedanib in combination with pemetrexed and cisplatin for chemotherapy-naive patients with advanced malignant pleural mesothelioma (LUME-Meso): a doubleblind, randomised, placebo-controlled phase 3 trial. Lancet Respir Med 2019; doi:10.1016/ S2213-2600(19)30139-0

Das Pleuramesotheliom ist eine seltene Erkrankung, die hauptsächlich durch eine Asbestexposition ausgelöst wird. Da der Krebs meist spät entdeckt wird und nicht mehr operiert werden kann, suchen Forscher nach Therapieoptionen. Eine Ergänzung der aktuell empfohlenen systemischen Chemotherapie um Nintedanib führte in ersten Studien zu längeren Überlebenszeiten. Scagliotti und Team haben jetzt eine entsprechende Phase-III Studie durchgeführt.

Das Pleuramesotheliom ist eine seltene, bösartige Lungenerkrankung, die in den meisten Fällen erst sehr spät erkannt wird und mit einer schlechten Prognose einhergeht. Auf pathogenetischer Ebene zählt eine Asbestexposition zu den wichtigsten Risikofaktoren. Und obgleich insbesondere in den Industrieländern das Risiko durch Asbest immer weiter zurückgegangen ist, steigt die Anzahl der Krankheitsfälle in Ländern mit niedrigem bis mittlerem Einkommen zurzeit messbar an. Aufgrund der späten Diagnose sind kurative Resektionen meist unmöglich. Betroffene Patienten werden als Alternative hauptsächlich mit einer systemischen Chemotherapie mit einer Kombination aus Pemetrexed und Cisplatin behandelt.

Da beim Wachstum des Pleuramesothelioms die Angiogenese einschließlich klassischer Faktoren wie VEGF und PDGF eine wichtige Rolle spielt, scheinen entsprechende Inhibitoren wie Nintedanib eine passende Therapieoption zu sein. Nachdem sich eine Ergänzung der Chemotherapie um diesen Angiogenesehemmer in ersten Phase-II-Studien bewehrt hatte und zu einem längeren Überleben führte, haben Scagliotti und sein
Team nun als nächsten Schritt eine entsprechende Phase-III-Studie durchgeführt.

Die sogenannte randomisierte, kontrollierte und doppelblinde LUME-Meso-Studie fand zwischen April 2016 und Januar 2018 an 120 Zentren in 27 Ländern statt. Geeignete Patienten erfüllten folgende Kriterien:

- Alter ab 18 Jahren,

- histologisch bestätigte Diagnose eines malignen Pleuramesothelioms,

- Malignom nicht resektabel,

- epitheloider Subtyp,

- Lebenserwartung von mindestens 3 Monaten,

- keine systemische Chemotherapie in der Anamnese.

Im nächsten Schritt ordneten die Forscher jeden Patienten einer von 2 Studiengruppen zu:

- Interventionsgruppe (IG): systemische Chemotherapie mit einer Kombination aus Pemetrexed und Cisplatin plus $200 \mathrm{mg}$ Nintedanib per os $2 \times$ täglich an den Tagen 2 bis 21 in jedem 21-Tage-Zyklus.

- Kontrollgruppe (KG): Chemotherapie wie in der Interventionsgruppe plus Placebo statt Nintedanib.

Jeder Patient sollte 6 Zyklen zu jeweils 21 Tagen mit $500 \mathrm{mg} / \mathrm{m}^{2}$ Pemetrexed und $75 \mathrm{mg} / \mathrm{m}^{2}$ Cisplatin intravenös als Infusion am ersten Tag erhalten. Als primären klinischen Endpunkt definierten die Autoren das progressionsfreie Überleben. Der wichtigste sekundärer Endpunkt war das Gesamtüberleben. Schließlich wollten die Forscher die Sicherheit von Nintedanib untersuchen und registrierten sämtliche Nebenwirkungen.

\section{Typische Nebenwirkungen}

541 Patienten erfüllten die Einschlusskriterien, 458 von ihnen nahmen schließlich an der Studie teil, jeweils 229 Patienten pro Studiengruppe. Sie waren im Durchschnitt 66 Jahre alt, 72\% (IG) bzw. 74\% (KG) von ihnen männlich, bei $62 \%$ bzw. $66 \%$ war eine Asbestexposition bekannt. Die mediane Behandlungsdauer lag bei 5,3 Monaten in der IG und bei 5,1 Monaten in der KG. Für das progressionsfreie
Überleben konnten die Forscher keinen signifikanten Unterschied zwischen den Gruppen feststellen. Auch für die sekundären Endpunkte waren die entsprechenden Ergebnisse der Studiengruppen vergleichbar.

Fast alle Patienten beider Gruppen entwickelten mindestens eine Nebenwirkung. Das am häufigsten berichtete unerwünschte Ereignis 3. Grades war eine Neutropenie bei 32\% der Patienten der IG und bei $24 \%$ der Patienten der KG. Schwerwiegende Nebenwirkungen wurden bei $44 \%$ der Patienten in der Nintedanib-Gruppe und bei 39\% der Patienten in der Placebo-Gruppe festgestellt. Das Nebenwirkungsprofil in der Interventionsgruppe entsprach den Erwartungen in Zusammenhang mit der Wirkstoffklasse von Nintedanib.

In ihrem Diskussionsteil stellen die Forscher fest, dass entgegen der Erwartung nach den Phase-II-Studien der primäre klinischer Endpunkt hier nicht erreicht werden konnte und sich zumindest in dieser Studie für die Ergänzung der systemischen Chemotherapie um Nintedanib kein Überlebensvorteil für die Patienten ergab. Es müsste - nach Meinung der Autoren - daher unbedingt nach weiteren Behandlungsoptionen gesucht werden.

\section{FAZIT}

In dieser randomisierten, doppelblinden Phase-III-Studie an Patienten mit inoperablem Pleuramesotheliom konnte eine Ergänzung der systemischen Chemotherapie um den Angiogenesehemmer Nintedanib das progressionsfreie Überleben im Vergleich zum Placebo nicht verlängern. Die Autorinnen/Autoren ziehen das Fazit, dass der primäre Endpunkt folglich nicht erreicht werden konnte und sehen die Notwendigkeit der weiteren Suche nach wirksamen Therapieoptionen für Patienten mit Pleuramesotheliomen.

Dipl.-Psych. Annika Simon, Hannover 